05

\title{
Влияние скорости нарастания тока на переходные процессы в сверхпроводниковом ограничителе тока
}

\author{
(ㄱ Д.Ф. Алферов, М.Р. Ахметгареев, Д.В. Евсин, И.Ф. Волошин, А.В. Калинов, \\ Л.М. Фишер, Е.В. Цхай
}

Акционерное общество Научно-исследовательский институт технической фризики и автоматизации (АО „НИИТФА“), 115230 Москва, Россия

e-mail: DFAlferov@niitfa.ru

(Поступило в Редакцию 22 апреля 2017 г.)

\begin{abstract}
Экспериментально исследовано влияние скорости нарастания тока до $1700 \mathrm{~A} / \mathrm{ms}$ на характеристики перехода блоков сверхпроводниковых (ВТСП) модулей из сверхпроводящего в нормальное состояние. Блоки отличались критическим током ВТСП ленты и конструктивным исполнением. Блоки ВТСП модулей используются в составе сверхпроводникового ограничителя тока (СОТ) резистивного типа для сетей переменного и постоянного токов. Полученные зависимости следует учитывать при проектировании резистивных СОТ.
\end{abstract}

DOI: 10.21883/JTF.2018.01.45477.2305

\section{Введение}

За годы существования высокотемпературных сверхпроводников (ВТСП) второго поколения их критические токи выросли на порядки, что позволяет говорить о реальных возможностях практического применения данного класса ВТСП материалов. Для этого необходимо знать физические свойства таких материалов, в частности критическую плотность тока $J_{C}$ и вольтамперные характеристики (BAX). Последние иллюстрируют переход ВТСП из сверхпроводящего состояния в нормальное. На ВАХ условно можно выделить три участка (стадии): сверхпроводящий (flux-creep), переходной (flux-flow) и нормальный (металлический) [1]. При плотностях транспортного тока ниже критического значения $J_{C}$ магнитное поле тока проникает в объем сверхпроводника в виде вихрей Абрикосова, которые в первом приближении неподвижны, поскольку удерживаются силами пиннинга (сверхпроводящее состояние). По мере роста плотности тока увеличивается сила Лоренца, действующая на вихрь, и при превышении критической плотности тока $J_{C}$ вихри начинают двигаться. Движение вихрей приводит к генерации электрического поля и появлению диссипации. Сверхпроводник переходит в резистивное состояние (переходное состояние), и электрическое поле в проводнике начинает быстро увеличиваться. Этот диссипативный процесс приводит к увеличению температуры сверхпроводника. Увеличение температуры ВТСП приводит к уменьшению критической плотности тока.

В этих состояниях ВАХ ВТСП обычно аппроксимируется степенной функцией

$$
E(J, T)=E_{C}\left(\frac{J}{J_{C}(T)}\right)^{\alpha},
$$

где $E_{C}=1 \mu V / \mathrm{cm}$ - критерий для определения критической плотности тока $J_{C}(T)$, которая зависит от темпе- ратуры сверхпроводника $T, \alpha-$ степенной показатель, который зависит от тока и температуры и изменяется для каждой стадии.

В сверхпроводящем состоянии плотность тока $J$ в сверхпроводнике меньше критической плотности тока, и сопротивление проводника очень мало. Это состояние характеризуется крутым участком ВАХ со степенным показателем $\alpha>30$ [2]. В узком диапазоне температур $T_{0}<T<T_{C}$ критическая плотность тока уменьшается линейно с увеличением температуры [3]

$$
J_{C}(T)=J_{C 0}\left(1-\frac{T-T_{0}}{T_{C}-T_{0}}\right)
$$

Здесь $T_{C}$ - критическая температура ВТСП ленты, $J_{C 0}$ - критическая плотность тока при температуре $T_{0}$. Когда температура ВТСП становится выше критической температуры $T>T_{C}$, сверхпроводник переходит в нормальное состояние, и начинается режим заметного ограничения роста тока.

Представленные выше характеристики ВТСП используются в резистивном сверхпроводниковом ограничителе тока (СОТ), который является принципиально новым электротехническим устройством, выполняющим защитную функцию в энергосистемах. При возникновении тока короткого замыкания (К3) в сети начинает возрастать ток $i$, скорость нарастания которого $d i / d t \sim U_{0} / L_{0}$ зависит от напряжения $U_{0}$ и индуктивности сети $L_{0}$. При превышении током $i$ критического тока $I_{C}=S J_{C}$, где $S$ - площадь сечения ВТСП ленты, сверхпроводник переходит из сверхпроводящего в резистивное состояние. Начинается режим ограничения тока за счет введения в сеть активного сопротивления ВТСП ленты, величина которого увеличивается с ростом тока. Быстрое увеличение сопротивления ВТСП ленты в резистивном режиме обусловлено как сильно нелинейной ВАХ сверхпроводника, так и последующим увеличением температуры 
ленты $T$ вследствие ее нагревания протекающим током и переходом в нормальное (металлическое) состояние.

Переходные характеристики такого устройства зависят от исполнения СОТ и материала ВТСП ленты. Скорость нагрева ВТСП ленты протекающим током увеличивается с увеличением скорости нарастания тока $d i / d t$ при возникновении К3 [4-6]. Соответственно должна уменьшаться длительность переходной стадии и изменяться ток перехода ВТСП в нормальное состояние. Эти основные параметры резистивного СОТ влияют на устойчивость ВТСП к аварийным воздействиям, что важно для практических применений. Однако анализ влияния $d i / d t$ на развитие переходного режима COT в настоящее время слабо отражен в литературе. Адекватное моделирование переходных процессов в таком устройстве является непростой задачей вследствие сильной нелинейности ВАХ и возможной неоднородности ВТСП лент.

В настоящей работе представлены результаты экспериментальных исследований переходных процессов в блоках ВТСП модулей СОТ при разных скоростях нарастания тока КЗ до $1700 \mathrm{~A} / \mathrm{ms}$. Такие режимы характерны для токов КЗ в сетях постоянного тока электрифицированного транспорта. Блоки отличались критическим током ВТСП ленты и конструктивным исполнением.

\section{Объект исследований}

Объектом исследований являлись блоки ВТСП модулей, предназначенные для использования в составе резистивного СОТ постоянного тока. Блоки размещались в криостате с жидким азотом при температуре $T=77 \mathrm{~K}$. Исследовались два блока ВТСП модулей Бл1 и Бл2, отличающихся ВТСП лентой и конструктивным исполнением (табл. 1).

Блок Бл1 содержит девять модулей: три последовательно соединенных звена, каждое из которых содержит три параллельно соединенных модуля [7]. Блок Бл2 содержит четыре последовательно соединенных ВТСП модуля. Сверхпроводниковые блоки Бл1 изготовлены из ВТСП ленты производства компании СуперОкс шириной $12 \mathrm{~mm}$. Подложка лент изготовлена

Таблица 1. Параметры блоков ВТСП модулей

\begin{tabular}{l|c|c}
\hline \multicolumn{1}{c|}{ Наименование } & Бл1 & Бл2 \\
\hline Производитель ВТСП ленты & СуперОкс & SuNAM \\
\hline Напряжение, $\mathrm{kV}$ & 3.5 & 4 \\
\hline Номинальный ток, А & 2000 & 2000 \\
\hline Число параллельных ВТСП лент & 12 & 4 \\
\hline Длина ВТСП ленты, $l_{b}, \mathrm{~m}$ & 31.5 & 40.8 \\
\hline Критический ток блока модулей, $I_{C b}, \mathrm{~A}$ & 3600 & 2800 \\
\hline Сопротивление блока при $T_{C}=93 \mathrm{~K}, \Omega$ & 0.26 & 1.22
\end{tabular}

Таблица 2. Параметры ВТСП модулей

\begin{tabular}{|c|c|c|}
\hline Наименование & M1 & M2 \\
\hline Производитель ВТСП ленты & СуперОкс & SuNAM \\
\hline Материал подложки & Hastelloy C276 & STS $310 \mathrm{~S}$ \\
\hline Длина ВТСП ленты, m & 10.5 & 10.2 \\
\hline Число параллельных ВТСП лент & 4 & 4 \\
\hline Критический ток ленты, $I_{C}$, А & 300 & 700 \\
\hline $\begin{array}{l}\text { Критический ток модуля, } \\
I_{C \bmod }, \mathrm{A}\end{array}$ & 1200 & 2800 \\
\hline $\begin{array}{l}\text { Сопротивление модуля при } \\
T_{0}=93 \mathrm{~K}, \Omega\end{array}$ & 0.26 & 0.305 \\
\hline
\end{tabular}

из сплава Hastelloy C276 толщиной $100 \mu \mathrm{m}$. Критический ток ВТСП ленты производства компании СуперОкс $I_{C 1}=300 \mathrm{~A}$.

Блок Бл2 изготовлен из ВТСП ленты производства компании SuNAM шириной $12 \mathrm{~mm}$. Подложка лент изготовлена из сплава STS $310 \mathrm{~S}$ толщиной $105 \mu \mathrm{m}$. Критический ток ВТСП ленты производства компании SuNAM $I_{C 2}=700$ A.

Сверхпроводниковые ленты покрыты слоем серебра толщиной $3 \mu \mathrm{m}$ и начинают переходить из сверхпроводящего в нормальное состояние при температуре $T_{C}=93 \mathrm{~K}$.

Каждый ВТСП модуль содержит шесть последовательно соединенных плоских элементов [7]. Элемент содержит четыре параллельные ВТСП ленты, наложенные бифилярно с двух сторон стеклотекстолитовой основы. Параметры модулей М1 и М2 представлены в табл. 2.

\section{Условия и методы исследований}

Принципиальная схема экспериментальной установки представлена на рис. 1. Установка состоит из конденсаторной батареи емкостью $C_{0} \approx 44 \mathrm{mF}$ на максимальное напряжение $6 \mathrm{kV}$ и реактора с индуктивностью $L_{0} \approx 2.6 \mathrm{mH}$. В качестве объекта исследований использовался блок ВТСП модулей $R_{2}$, последовательно соединенный с быстродействующим вакуумным выключателем (БВВ) (очерчен штриховой линией). БВВ содержит вакуумную дугогасительную камеру $(Q)$, контур противотока $\left(L_{1}, C_{1}, Q_{1}\right)$ и ограничитель перенапряжений $R_{1}$ [8]. Образец исследований подключался к предварительно заряженной конденсаторной батарее с помощью разрядника $Q_{2}$. Шунтирующий разрядник $Q_{3}$ предназначен для защиты исследуемого объекта в случае отказа выключателя.

Перед каждым испытанием контакты выключателя были замкнуты. После включения разрядника $Q_{2}$ в цепи начинал протекать разрядный ток $i$, амплитуда $I_{m}$ и 


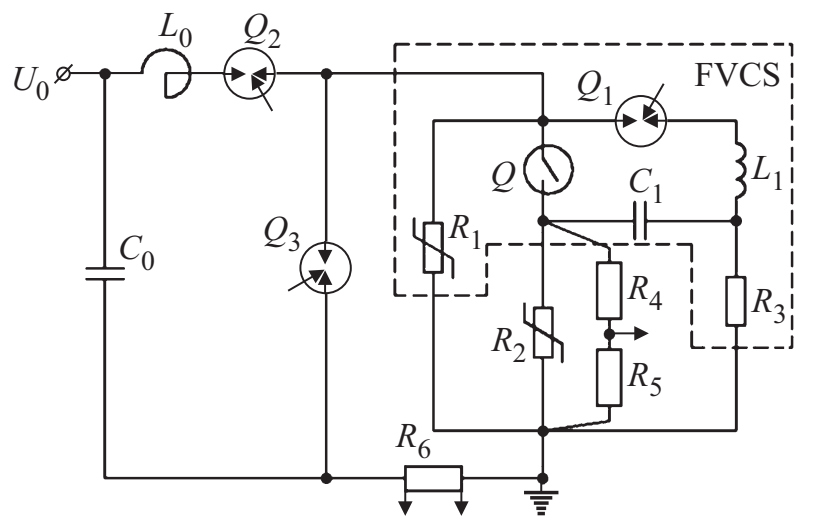

Рис. 1. Схема испытаний.

максимальная скорость нарастания $(d i / d t)_{\max }=U_{0} / L_{0}$ которого задавались уровнем зарядного напряжения $U_{0}$.

Измерение тока $i$ производилось с помощью шунта с сопротивлением $R_{6}=0.154 \mathrm{~m} \Omega$. Напряжение на исследуемом объекте $R_{2}$ измерялось с помощью омического делителя $R_{4}-R_{5}$ с коэффициентом деления 221. Электрические сигналы с датчиков тока и напряжения одновременно регистрировались на осциллографе Tektronix DPO 4034 с последующим сохранением и обработкой на персональном компьютере.

По значениям напряжения и тока через исследуемый объект определялась величина сопротивления $R_{2}(t)$ в режиме ограничения тока до момента его отключения вакуумным выключателем. Зависимость $R_{2}(t)$ использовалась для оценки температуры ВТСП ленты [7].

\section{Результаты исследований блоков ВТСП модулей}

Первая серия исследований в режиме имитации короткого замыкания проводилась на блоке Бл1 (табл. 1) с ВТСП лентой (СуперОкс), $I_{\mathrm{cb} 1}=3600$ А. Зарядное напряжение батареи конденсаторов $U_{0}$ изменялось от 2600 до $4000 \mathrm{~V}$.

Зависимости тока $i(t)$ и напряжения $U(t)(a)$ от времени $t$ и найденные из этих зависимостей изменения сопротивления блока ВТСП модулей $(b)$ для разных $(d i / d t)_{\max }$ представлены на рис. 2.

Вторая серия исследований в режиме имитации короткого замыкания проводилась на блоке Бл2 (табл. 1) с ВТСП лентой (SuNAM), $I_{\mathrm{cb} 2}=2800$ А. Зарядное напряжение батареи конденсаторов $U_{0}$ изменялось от 2000 до $4100 \mathrm{~V}$. Зависимости тока $i(t)$, напряжения $U(t)(a)$ от времени $t$ и найденные из этих зависимостей изменения сопротивления блока Бл2 $(b)$ для разных $(d i / d t)_{\max }$ представлены на рис. 3 .

Из рис. 2 и 3 найдены ВАХ блоков ВТСП модулей Бл1 и Бл2 для разных $(d i / d t)_{\max }$ (рис. 4).

\section{1. Анализ результатов измерений}

Из рис. 2 и 3 видно, что изменения тока и напряжения во времени в разных блоках ВТСП модулей (Бл1 и Бл2) имеют общие тенденции. С ростом тока $i$ ВТСП сохранял сверхпроводимость вплоть до достижения критического тока $i=I_{\mathrm{cb} 1}=3600 \mathrm{~A}$ (рис. 2, $a$ ) и $i=I_{\mathrm{cb} 2}=2800 \mathrm{~A}$ (рис. 3,a). В этом интервале изменения тока скорость роста тока $d i / d t=(d i / d t)_{\max }$ можно считать постоянной для каждого опыта. При превышении критического тока ВТСП начинал переходить из сверхпроводящего в переходное резистивное состояние, что проявлялось в виде появления напряжения на блоке ВТСП модулей. С увеличением $(d i / d t)_{\max }$ наблюдался сдвиг кривых напряжения в сторону меньших времен, т. е. быстрее достигалось критическое значение тока. Это отражалось на BAX (рис. 4) в виде смещения кривых 1-3 (Бл1) и кривых 4-8 (Бл2) в сторону больших токов, т.е. при одном и том же напряжении на блоке ВТСП модулей ток перехода увеличивался.

Форма изменения напряжения на блоке ВТСП модулей характеризуется участком с быстрым увеличением крутизны роста напряжения $d U / d t$ при $i>I_{\mathrm{cb} 1}=3600 \mathrm{~A}$ (рис. 2,a) и при $i>I_{\mathrm{cb} 2}=2800 \mathrm{~A}$ (рис. 3,a) с последу-
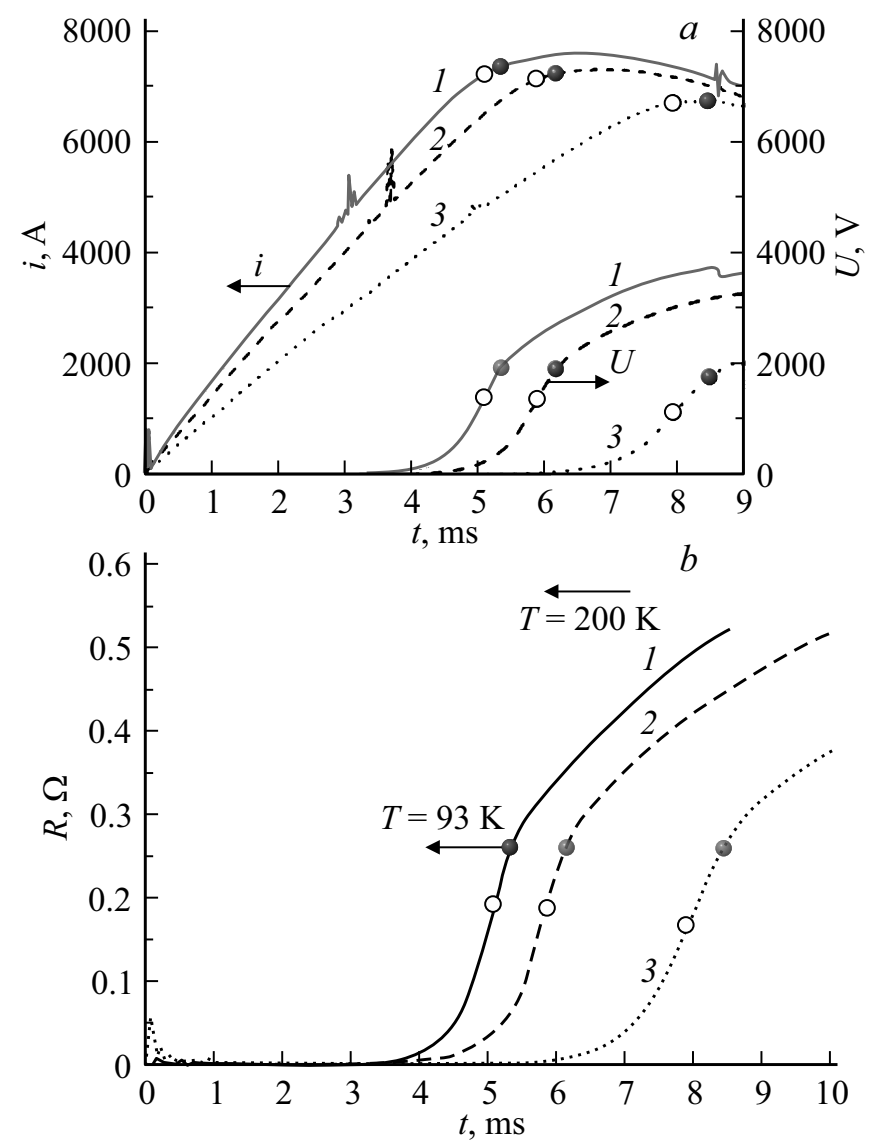

Рис. 2. Изменения напряжения $U(t)$ и тока $i(t)-(a)$, и сопротивления $R(t)-(b)$ блока ВТСП модулей Бл1 при разных $(d i / d t)_{\max }: 1-1670,2-1440,3-1007 \mathrm{~A} / \mathrm{ms}$ 

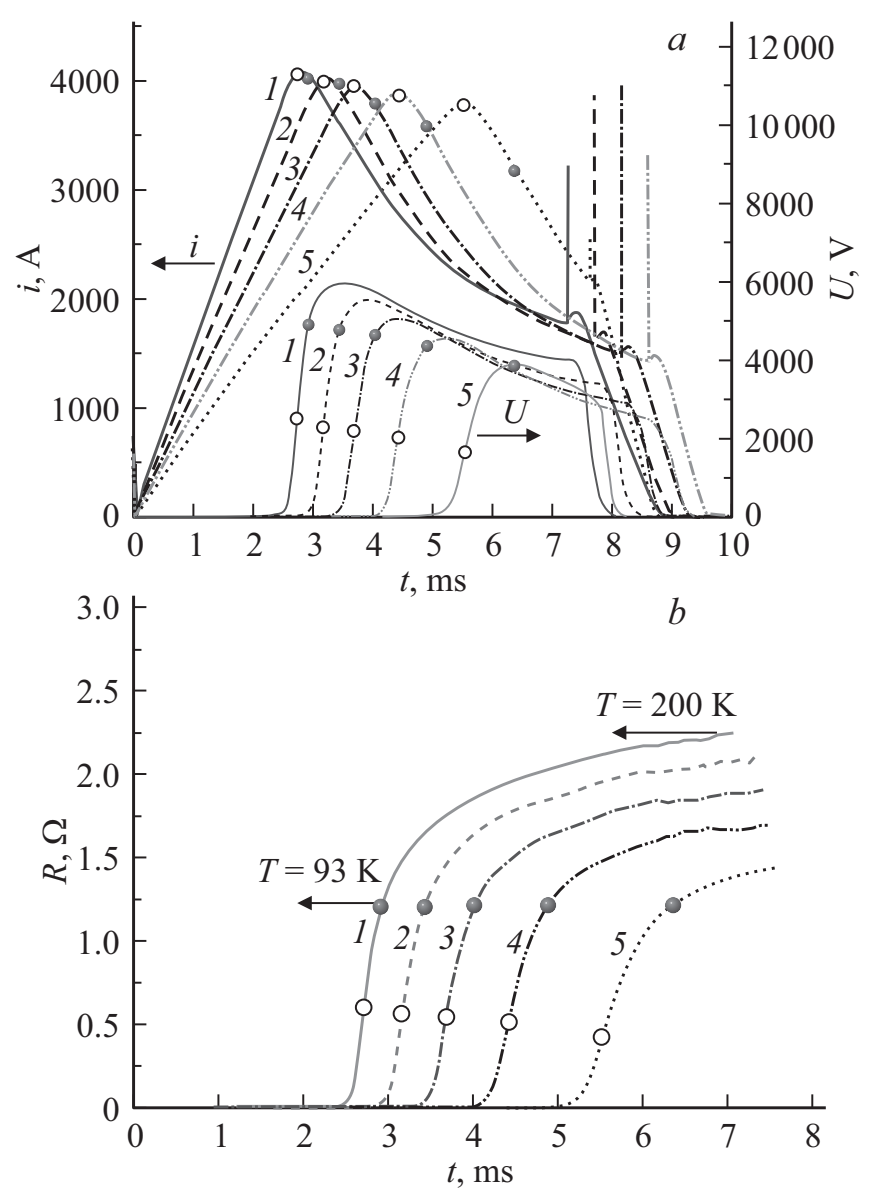

Рис. 3. Изменения напряжения $U(t)$ и тока $i(t)-(a)$, и сопротивления $R(t)-(b)$ блока ВТСП модулей Бл2 при разных $(d i / d t)_{\max }: 1-1570,2-1340,3-1150,4-970$, $5-760 \mathrm{~A} / \mathrm{ms}$.

ющим заметным замедлением роста напряжения. Аналогичный перегиб наблюдался и в зависимости сопротивления $R(t)$ блока ВТСП модулей $R=U / i$ (рис. $2, b, 3, b)$.

Участок кривой с крутым ростом напряжения определим как процесс перехода ВТСП из сверхпроводящего состояния в резистивное состояние (flux-flow). Этот переходный процесс начинал заметно проявляться (быстрый рост напряжения и сопротивления), когда сверхпроводник нагревался протекающим током до температуры $T>77 \mathrm{~K}$, и завершался в момент $t_{n}$ при нагреве ВТСП до температуры $T_{C} \approx 93 \mathrm{~K}$. При $T \approx 93 \mathrm{~K}$ сопротивление блока модулей Бл1 равно сопротивлению одного модуля и составляет $R_{n 1} \approx 0.26 \Omega$ (рис. $2, b$ ), а сопротивление блока модулей Бл 2 , составляет $R_{n 2} \approx 1.22$ или $0.305 \Omega$ на один модуль (рис. 3, $b$ ). На рис. 2-4 сплошными кружками $(\bullet)$ помечены ток $i_{n}$, напряжение $U_{n}$ и сопротивление $R_{n}$ в момент $t_{n}$ перехода ВТСП в нормальное состояние при разных $(d i / d t)_{\max }$.

Положим, что стадия перехода начинается в момент перегиба в зависимостях $U(t)$ и $R(t)$. Точку перегиба можно найти путем дифференцирования кривой $U(t)$ и определения момента $t_{t}$ максимума полученной за- висимости $d U / d t$. Этот момент определим как начало переходного процесса. Мгновенные значения тока $i_{t}$, напряжения $U_{t}$ и сопротивления $R_{t}$ в момент $t_{t}$ при разных значениях $(d i / d t)_{\max }$ показаны пустыми кружками (о) на рис. $2-4$. При $t \geq t_{t}$ рост тока прекращался и ток начинал медленно спадать вследствие уменьшения степенного показателя ВАХ до единицы и увеличения сопротивления ВТСП в результате его джоулева разогрева. В отличие от Бл1 напряжение $U_{t}$ в точках перегиба ВАХ в Бл2 заметно увеличивался с увеличением $(d i / d t)_{\max }$ (рис. 4).

Время до начала перехода $t_{t}$ и до момента $t_{n}$ завершения перехода ВТСП в нормальное состояние в блоках Бл1 $\left(t_{t 1}, t_{n 1}\right)$ и Бл2 $\left(t_{t 2}, t_{n 2}\right)$ уменьшалось с увеличением скорости роста тока $t_{t} \sim t_{n} \sim 1 /(d i / d t)_{\max }$ (рис. 5). Для каждого $(d i / d t)_{\max }$ переходная стадия в Бл1 начиналась в 2 раза позже, чем в Бл2 $\left(t_{t 1} / t_{t 2} \approx 2\right)$. Длительность переходной стадии $t_{n}-t_{t}$ в обоих блоках

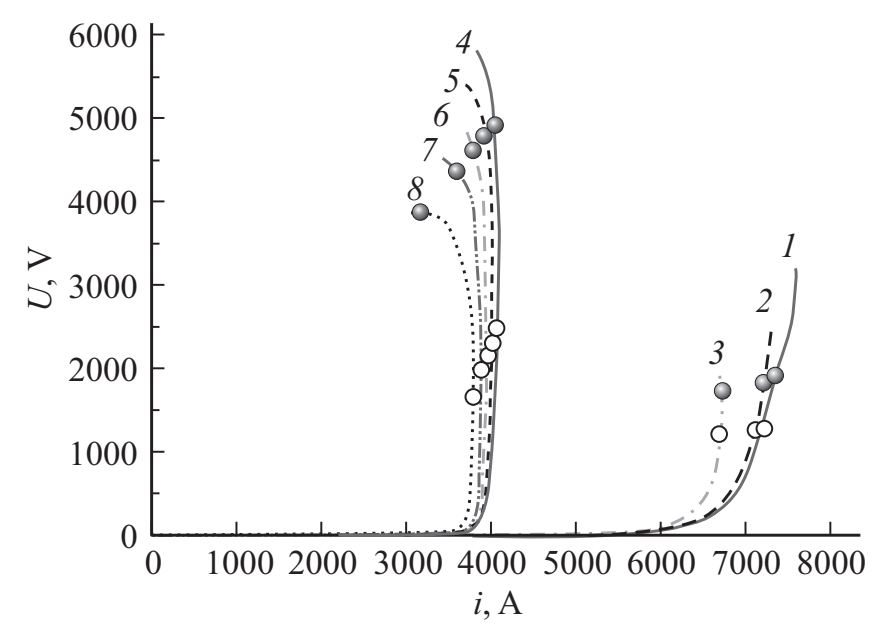

Рис. 4. ВАХ блоков ВТСП модулей при разных $(d i / d t)_{\max }$ : Бл1: $1-1670,2-1440,3-1007$, Бл2: $4-1570,5-1340$, $6-1150,7-970,8-760 \mathrm{~A} / \mathrm{ms}$.

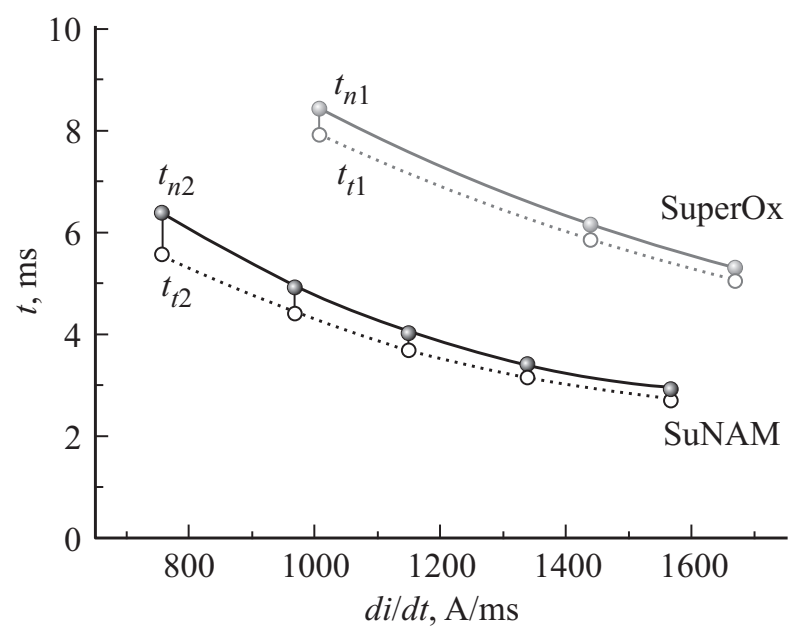

Рис. 5. Зависимости моментов перехода $t_{t}$ и $t_{n}$ от $(d i / d t)_{\max }$ в блоке Бл1 $\left(t_{t 1}, t_{n 1}\right)$ и в блоке Бл2 $\left(t_{t 2}, t_{n 2}\right)$. 


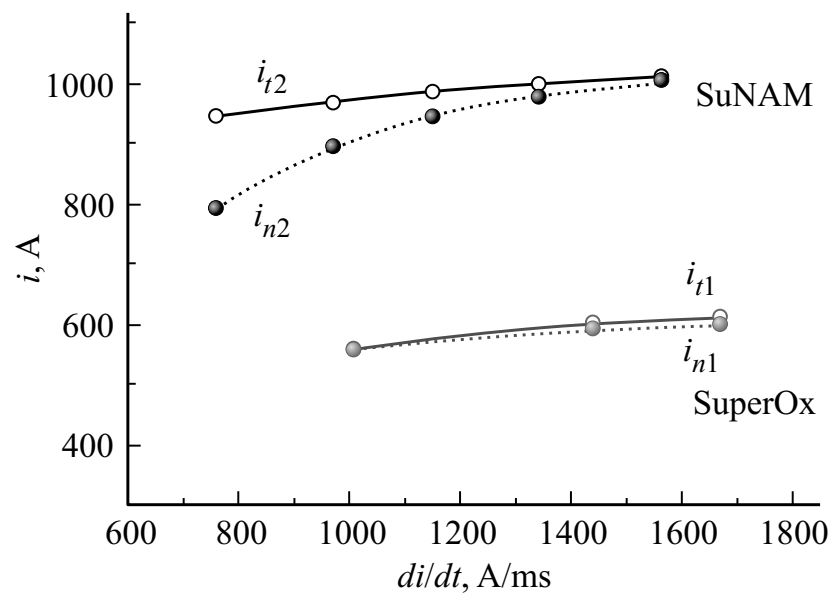

Pис. 6. Зависимости токов $i_{t}$ и $i_{n}$ в блоках Бл1 и Бл2 от $(d i / d t)_{\max }$.

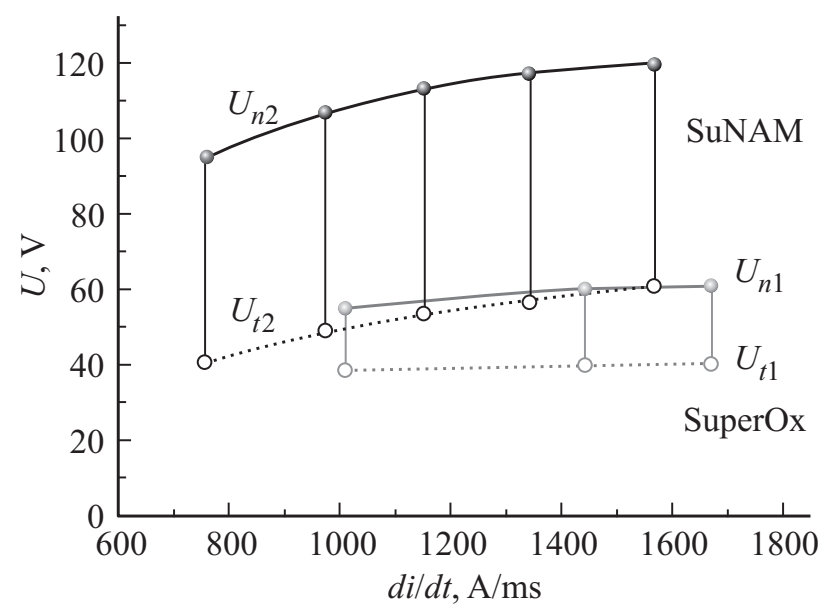

Рис. 7. Зависимости напряжений $U_{t}$ и $U_{n}$ на блоках Бл 1 и Бл2 от $(d i / d t)_{\max }$.

уменьшалась с увеличением $(d i / d t)_{\max }$ примерно одинаково.

На рис. 6 представлены зависимости от $(d i / d t)_{\max }$ токов перехода $i_{t 1} / m_{1}$ и $i_{n 1} / m_{1}\left(m_{1}=12\right)$ в блоке модулей Бл1 и токов перехода $i_{t 2} / m_{2}$ и $i_{n 2} / m_{2}\left(m_{2}=4\right)$ в блоке модулей Бл2, нормированных на одну ВТСП ленту. В блоке модулей Бл 1 ток $i_{t 1}$ в 2 раза превышал критический ток $I_{\mathrm{cb} 1}$ и мало отличался от тока $i_{n 1}$ в момент завершения перехода в нормальное состояние. С увеличением $(d i / d t)_{\max }$ в 1.5 раза ток $i_{t 1}$ увеличился на 7\%. В блоке модулей Бл2 ток $i_{t 2}$ в момент начала переходной стадии в 1.35 раза превышал критический ток $I_{\mathrm{cb} 2}$ при $(d i / d t)_{\max }=760 \mathrm{~A} / \mathrm{ms}$ и увеличился на $7 \%$ при увеличении $(d i / d t)_{\max }$ в 2 раза. При этом ток $i_{n 2}$ в момент перехода ВТСП в нормальное состояние увеличился на $21 \%$ и приблизился к току $i_{t 2}$.

Зависимости удельных напряжений $U_{t 1} / l_{b 1}$ в момент $t_{t 1}$ и $U_{n 1} / l_{b 1}$ в момент $t_{n 1}$ на блоке модулей Бл1 и удельных напряжений $U_{t 2} / l_{b 2}$ в момент $t_{t 2}$ и $U_{n 2} / l_{b 2}$ в момент $t_{n 2}$ на блоке модулей Бл2 представлены на рис. 7.
При увеличении $(d i / d t)_{\max }$ в 1.5 раза удельное напряжение $U_{t 1} / l_{b 1}=(28-39) \mathrm{V} / \mathrm{m}$ в момент $t_{t 1}$ на блоке Бл1 практически не менялось, а удельное напряжение $U_{n 1} / l_{b 1}$ в момент $t_{n 1}$ завершения перехода в нормальное состояние увеличилось на $10 \%$. В блоке Бл2 при увеличении $(d i / d t)_{\max }$ в 2 раза удельное напряжение $U_{t 2} / l_{b 2}$ увеличилось на $33 \%$, а удельное напряжение $U_{n 2} / l_{b 2}$ увеличилось на 20\%. Интервал $t_{n}-t_{t}$ между моментом $t_{t}$ начала перехода и моментом $t_{n}$ завершения перехода ВТСП в нормальное состояние уменьшался с увеличением $(d i / d t)_{\max }$ от 0.52 до $0.25 \mathrm{~ms}$ в блоке Бл1 и от 0.84 до $0.2 \mathrm{~ms}$ в блоке Бл2.

В момент отключения тока сопротивление блока ВТСП модулей Бл1 увеличивалось до $0.6 \Omega$, а блока ВТСП модулей Бл2 до $2.2 \Omega$, что соответствует нагреву ВТСП ленты до температуры порядка $200 \mathrm{~K}$ (рис. 2, $b$ и $3, b)$.

\section{Заключение}

Исследованы характеристики перехода блоков ВТСП модулей из сверхпроводящего в нормальное состояние при разных скоростях нарастания тока $(d i / d t)_{\max }$ до $1700 \mathrm{~A} / \mathrm{ms}$. Сверхпроводниковые блоки изготовлены из ВТСП лент разных производителей: производства компании СуперОкс (Бл1) с критическим током $I_{c 1}=300 \mathrm{~A}\left(I_{C b 1}=3600 \mathrm{~A}\right)$ и компании SuNAM (Бл2) с критическим током $I_{c 2}=700 \mathrm{~A}\left(I_{C b 1}=2800 \mathrm{~A}\right)$. Переходная стадия начиналась в момент $t_{t}$ перегиба кривой напряжения на блоке ВТСП модулей и завершалась в момент $t_{n}$ при нагреве ВТСП до критической температуры $T_{C}=93 \mathrm{~K}$.

В результате исследований установлено, что с увеличением $(d i / d t)_{\max }$

- уменьшается время от начала тока до момента $t_{t}$ начала перехода ВТСП в нормальное состояние $t_{t} \sim 1 /(d i / d t)_{\max }$, причем момент $t_{t 1}$ в блоке Бл1 наступал в 2 раза позже, чем момент $t_{t 2}$ в блоке Бл2;

- уменьшается интервал $t_{n}-t_{t}$ между моментом $t_{t}$ начала переходной стадии и моментом tn завершения полного перехода ВТСП в нормальное состояние, причем это уменьшение не зависело от исполнения блока ВТСП модулей;

- увеличивается ток $i_{t}$ начала перехода и ток $i_{n}$ завершения перехода в нормальное состояние, причем увеличение тока $i_{n}$ в блоке Бл2 более заметно по сравнению с блоком Бл2, что может быть обусловлено более быстрым спадом тока на переходной стадии после достижения тока $i_{t}$;

- увеличивается напряжение перехода $U_{t 2}$ в блоке Бл2, а в блоке Бл1 напряжение $U_{t 1}$ остается практически постоянным;

- увеличивается приращение напряжения $U_{n}-U_{t}$ в течение переходной стадии, причем это приращение в блоке Бл2 в 2 раза превышает соответствующее приращение в блоке Бл1. 
Из полученных результатов следует, что с увеличением критического тока влияние скорости нарастания тока на характеристики переходной стадии блоков ВТСП модулей становится более существенным.

Полученные зависимости следует учитывать при проектировании резистивных СОТ.

\section{Список литературы}

[1] De Sousa W.T.B., Polasek A., Silva F.A., Dias R., Jurelo A.R., De Andrade R. // Jr. Simulations, Tests of MCP-BSCCO-2212 Superconducting Fault Current Limiters //IEEE Trans Appl. Superconductiv. 2012. Vol. 22. N 2.

[2] Degtyarenko P.N., Dulkin I.N., Fisher L.M., Kalinov A.V., Voloshin I.F., Yampolsky V.A. // Low Temp. Phys. 2011. Vol. 37. P. 101-106.

[3] Lee W.S., Nam S., Kim J., Lee J., Ko T.K. // IEEE Trans Appl. Superconductiv. 2015. Vol. 25. N 3.

[4] Alferov D., Budovsky A., Dul'kin I., Fisher L., Ivanov V., Sidorov V., Shul'ga R., Tshay E., Yevsin D. // Proc. of the IEEE/CSC and ESAS European Superconductivity News Forum ESNF. 2010. N 11. P. 1-8.

[5] Алферов Д.Ф., Ахметгареев М.Р., Будовский А.И., Бунин Р.А., Евсин Д.В., Иванов В.П., Сидоров В.А., Цхай Е.В. // Электричество. 2012. № 9. С. 12-22.

[6] Алферов Д.Ф., Ахметгареев М.Р., Будовский А.И., Бунин Р.А., Волошин И.Ф., Дегтяренко П.Н., Евсин Д.В., Иванов В.П., Сидоров В.А, Фишер Л.М., Цхай Е.В. // Известия АН. Энергетика. 2011. № 4. С. 30-36.

[7] Фишер Л.М., Алферов Д.Ф., Ахметгареев М.Р., Будовский А.И., Евсин Д.В., Волошин И.Ф., Калинов А.В. // Ядерная физика и инжиниринг. 2015. Т. 6. № 11-12. C. $553-561$.

[8] Алферов Д.Ф., Ахметгареев М.Р., Будовский А.И., Евсин Д.В., Фишер Л.М. // Электро. 2015. № 3. С. 43-47. 